\title{
ESTUDO QUASE-EXPERIMENTAL SOBRE ARGUMENTAÇÃO EM SITUAÇÕES DE DILEMA EM DIFERENTES CONDIÇÕES DE INTERAÇÃO
}

\author{
Nadia Peralta ${ }^{\mathrm{i}}$ \\ Néstor Rosellii
}

Resumo: Este trabalho aborda a argumentação entendida como o produto do confronto de pontos de vista em um contexto de interação sociocognitiva. Propõe-se um estudo quase-experimental no qual sujeitos em duplas discutiram sobre seus argumentos com respeito a uma situação de dilema de acordo com duas modalidades: livre (sem pautas de interação) ou assistida (pelo pesquisador). $\mathrm{Na}$ modalidade assistida, a intervenção do pesquisador teve como objetivo reforçar a interação para melhorar a qualidade argumentativa. Foram avaliadas tanto as intervenções dos sujeitos durante as interações quanto os escritos individuais realizados após a interação. Os resultados mostram avanços nas duplas assistidas no que diz respeito às três dimensões avaliadas: estilo argumentativo, competência argumentativa e dialogicidade.

Palavras-chave: Argumentação. Interação Sociocognitiva. Dialogicidade. Competência Argumentativa.

Abstract: This paper approaches argumentation understood as the product of the confrontation of points of view in a context of sociocognitive interaction. We propose a quasi-experimental study in which subjects in dyads discussed their arguments regarding a dilemmatic situation according to two modalities: free (without patterns of interaction) or assisted (by the researcher). In the assisted modality, the intervention of the researcher aimed to prop up the interaction to improve the argumentative quality. Both the recordings of the interactions and the writings of the subjects performed after the interaction were evaluated. The results show advances in assisted dyads in terms of the three dimensions evaluated: argumentative style, argumentative competence and dialogic interaction

Keywords: Argumentation. Sociocognitive interaction. Dialogic interaction. Argumentative competence.

\footnotetext{
' Professora Doutora da Faculdade de Psicologia da Universidade Nacional de Rosario (UNR) e pesquisadora do CONICET, Argentina.E-mail: nadiasoledadperalta@gmail.com.

ii Professor Doutor da Faculdade de Psicologia e Psicopedagogia da Pontifícia Universidade Católica Argentina (UCA), Argentina. E-mail: nestorroselli@uca.edu.ar.
} 
EID\&A - Revista Eletrônica de Estudos Integrados em Discurso e Argumentação, llhéus, n. 15, jan./jun.2018.

\section{Introdução}

No âmbito universitário, a argumentação é considerada uma importante e esperada habilidade nos estudantes, além de ter uma natureza conflitiva, já que é, em essência, produto do confronto de pontos de vistas em um processo de interação sociocognitiva. Essa perspectiva interacionista compreende a argumentação como uma atividade psicossocial que beneficia os sujeitos em termos cognitivos, permitindo-os avançar em suas aprendizagens (FELTON; KUHN, 2001).

Nesse contexto, parte-se do princípio socioconstrutivo que considera o diálogo argumentativo como o cenário ideal para a construção do conhecimento (PEREZ-ECHEVERRÍA; POSTIGO; GARCIA-MILÁ, 2016) e busca-se evidenciar o valor das situações interativas acima das individuais e o valor da argumentação acima do que não é argumentativo (PERALTA, 2012; PERALTA; ROSELLI; BORGOBELLO, 2012; PERALTA; ROSELLI, 2017).

Contudo, segundo Leitão (2000), a argumentação pode ser entendida como um modo racional de enfrentar um conflito que requer, por um lado, a produção de um discurso para definir e justificar uma posição, e, por outro, supõe que aqueles que argumentam verifiquem suas posições à luz das posições dos outros, o que os envolve em um processo social com finalidade e contexto determinados.

Nesse contexto, considera-se fundamental estudar a argumentação de estudantes universitários do ponto de vista interacionista por meio de desenhos experimentais que permitam fornecer dados empíricos para elucidar seus possíveis benefícios. Por esse motivo, este trabalho se centrou no estudo do estilo, na competência e na dialogicidade argumentativa em diferentes dimensões de interação; duplas com e sem assistência (andaimagem da dialogicidade) do pesquisador e dos indivíduos, e também de maneira oral durante a interação ou a produção escrita individual, em tarefas que apresentam situações de dilema.

Existe uma diversidade de formas de estudar a argumentação, desde perspectivas centradas na análise formal até a análise do conteúdo. Neste trabalho, compreende-se que a argumentação, como fenômeno social interativo, pode ser assistida (andaimada), apresentar-se no contexto de um diálogo ou ser produto de uma pessoa, e ser estudada em suas três dimensões: estilo (SANTIBÁÑEZ, MIGDALEK, ROSEMBERG, 2016), 
EID\&A - Revista Eletrônica de Estudos Integrados em Discurso e Argumentação, Ilhéus, n. 15, jan./jun.2018.

competência (CRUZ AGUILAR, CARMONA FERNÁNDEZ, 2014) e dialogicidade (PERALTA, ROSELLI, BORGOBELLO, 2012).

A definição de estilo faz referência ao modo como o sujeito oferece ao interlocutor razões com determinada qualidade. Essa qualidade depende da relevância, aceitabilidade, da apresentação de dados, fatos ou valores em relação ao tema e seu vínculo com o afetivo (SANTIBÁÑEZ, MIGDALEK, ROSEMBERG, 2016).

Por outro lado, a competência faz referência aos aspectos cognitivos vinculados com a argumentação, ou seja, a argumentação como atividade mental que envolve outras capacidades (SANTIBÁÑEZ, 2015). Tal competência tem sido estudada em diferentes contextos, fundamentalmente no âmbito universitário, porque é considerada como a forma de medir a capacidade dos jovens para participar de atividades que supõem a tomada de decisão, emissão de julgamentos ou a tomada de posição diante de uma situação profissional. Nesse sentido, Guzmán Cedillo, Flores Macías e Tirado Segura (2012) realizaram um estudo com o objetivo de desenvolver uma rubrica para avaliar as mensagens em fóruns de discussão online. Em sua elaboração, levaram em conta uma análise integral dos recursos, avaliando a estrutura, sintaxe, transição discursiva, atitude e análise da evidência enunciada, considerando esta rubrica de sucesso para a avaliação da competência argumentativa. GarcíaBarrera (2015) chegou a resultados semelhantes em um estudo no qual utilizou o role play para o desenvolvimento da capacidade argumentativa em docentes em formação na modalidade de educação à distância.

Nessa mesma linha, Cruz e Carmona (2014) construíram um instrumento composto por respostas abertas para detectar competências argumentativas. Os autores propuseram, como categorias operacionais da competência, a capacidade de emitir um enunciado, contra-argumentar e, finalmente, refutar.

Por último, com dialogicidade, enfatizam-se os aspectos interativos da argumentação, ou seja, a participação, a avaliação e a contra-argumentação. Em linhas gerais, considera-se que é possível, diante de uma intervenção concreta, estimular a participação simétrica dos participantes, a avaliação isto é, a capacidade dos sujeitos para expressar acordos e desacordos com as opiniões dos companheiros - e a contra-argumentação - em outras palavras, a capacidade de levar em conta o ponto de vista alternativo ao seu próprio (PERALTA, ROSELLI, BORGOBELLO, 2012; PERALTA, ROSELLI, 2017). 
EID\&A - Revista Eletrônica de Estudos Integrados em Discurso e Argumentação, llhéus, n. 15, jan./jun.2018.

A respeito da tarefa de tomada de decisão em situações de dilema, Achury e Álvarez Hoyos (2015) realizaram um estudo no qual se desenvolveu, com estudantes do ensino médio de uma escola de Bogotá, uma sequência didática que incluiu uma problemática sociocientífica sobre Química. Segundo os autores, a natureza controversa da problemática possibilitou que os estudantes apresentassem altos níveis de argumentação na tomada de decisão, já que levantaram questionamentos, implementaram qualificadores e utilizaram informação para manter suas posiciones.

Por fim, um antecedente direto desse estudo é o trabalho de Peralta e Roselli (2017), os quais analisaram, a partir de um desenho experimental, tipos de modalidades argumentativas em função de diferentes tamanhos de grupos (duplas e trios) e diferentes tipos de tarefas (tarefas lógicas e de conhecimento objetivo e tarefas de opinião ou subjetivas), entre elas, tarefas de tomada de decisão a respeito das situações morais de dilema. Os resultados mostraram que, nesse tipo de tarefa, predomina a resolução argumentativa individual, ou seja, o emprego de argumentações por parte dos sujeitos envolvidos em uma interação conflitiva cujo único objetivo é fortalecer a própria opinião contra a do outro, sem que isso evolua para um consenso intersubjetivo.

\section{Método}

\subsection{Desenho}

O dispositivo empírico utilizado consistiu-se em um desenho quaseexperimental, garantindo o controle de certas variáveis, mas, ao mesmo tempo, recorreu-se à análise dos processos discursivos dos sujeitos.

\subsection{Participantes}

A amostra foi constituída por 48 sujeitos, todos estudantes do primeiro ano de diversos cursos da área das ciências sociais ${ }^{1}$ de universidades públicas da Argentina; 24 formaram o grupo experimental (trabalho em duplas

\footnotetext{
${ }^{1}$ N.T.: Em espanhol, ciencias sociales pode se referir ao conjunto de disciplinas acadêmicas que estudam a origem e o desenvolvimento da sociedade, das instituições e das relações e ideias que configuram a vida social. Nesse sentido, as ciências sociais incluiriam disciplinas relacionadas com a interação social, o sistema cognitivo humano, a evolução das sociedades e a aplicação desses saberes se formam, como a Antropologia, Arqueologia, Sociologia, Ciências Políticas, Economia, Geografia, História e Historiografia, Direito, Psicologia, Criminologia e Psicologia Social.
} 
EID\&A - Revista Eletrônica de Estudos Integrados em Discurso e Argumentação, Ilhéus, n. 15, jan./jun.2018.

assistidas) e 24 constituíram o grupo de controle (trabalho em duplas livres). Todos os sujeitos participaram voluntariamente da pesquisa, assinaram o termo de consentimento e foram informados sobre o uso anônimo dos dados.

\subsection{Materiais}

Uma tarefa foi construída na qual os sujeitos deveriam definir que posição tomar diante de quatro situações de dilema e podiam optar por quatro possibilidades: concordar ou não com uma determinada posição e duas possibilidades intermediárias, uma mais próxima do acordo e outra mais vinculada ao desacordo.

\subsection{Procedimento}

Todos os sujeitos realizaram um pré-teste que consistia de 4 situações de dilema, que requeriam apenas respostas sem argumentação para detectar suas orientações de base. O objetivo foi detectar as orientações de base dos sujeitos a fim de selecionar os dois subtipos da tarefa, o que permitiria constituir as duplas heterogêneas do estudo.

\subsection{Situação experimental}

Os sujeitos das duplas discutiram sobre seus argumentos a respeito da situação de dilema segundo duas modalidades: livre (sem pautas de interação) ou assistida (pelo pesquisador). No caso das duplas assistidas, a intervenção do pesquisador teve como objetivo reforçar a interação para melhorar a qualidade argumentativa, intervindo especificamente em três aspectos:

- a participação (estimular a dar uma opinião e escutar a do companheiro);

- a avaliação (consultar se os sujeitos estavam de acordo ou não com o que foi exposto pelo outro sujeito);

- a contra-argumentação (levantar um argumento alternativo àquele oferecido pelo companheiro).

Uma vez finalizada a fase experimental, todos os sujeitos realizaram dois pós-testes. O primeiro pós-teste consistiu em uma discussão não assistida de uma situação de dilema sobre a qual as duplas não discutiram na sessão experimental. Esse pós-teste teve como objetivo medir a melhoria na 
EID\&A - Revista Eletrônica de Estudos Integrados em Discurso e Argumentação, Ilhéus, n. 15, jan./jun.2018.

dialogicidade argumentativa (dinâmica da interação) entre os sujeitos que participaram de duplas assistidas e os sujeitos que trabalharam livremente sem tal assistência.

No segundo pós-teste, os sujeitos das duplas expressaram por escrito (individualmente) as argumentações da situação de dilema que realizaram oralmente na condição experimental (dialógica). Esse pós-teste teve como objetivo medir a melhoria na competência argumentativa individual entre os sujeitos que participaram de duplas assistidas e os que não passaram por tal situação.

Em síntese, com o primeiro pós-teste, foi avaliada a dialogicidade a partir de critérios formais e, com o segundo pós-teste, foi testada a qualidade da competência argumentativa em função de critérios de conteúdo.

Tabela 1 - Desenho geral da pesquisa

\begin{tabular}{|c|c|c|c|}
\hline Pré-teste & $\begin{array}{c}\text { Situação } \\
\text { Experimental }\end{array}$ & $\begin{array}{c}\text { Pós-teste } 1 \\
\text { Oral }\end{array}$ & $\begin{array}{c}\text { Pós-teste } 2 \\
\text { Escrito }\end{array}$ \\
\hline $\begin{array}{l}\text { Individual não } \\
\text { argumentativo } \\
\text { (apenas resposta) }\end{array}$ & $\begin{array}{l}\text { Grupo experimental } \\
\text { Discussão dialógica } \\
\text { argumentativa } \\
\text { assistida da } \\
\text { situação de dilema } 1 \\
\text { (12 duplas) } \\
\text { Grupo controle } \\
\text { Discussão dialógica } \\
\text { argumentativa não } \\
\text { assistida da } \\
\text { situação de dilema } 1 \\
\text { (12 duplas) }\end{array}$ & $\begin{array}{l}\text { Discussão dialógica } \\
\text { argumentativa } \\
\text { não assistida de } \\
\text { situação de dilema } 2\end{array}$ & $\begin{array}{c}\text { Argumentação } \\
\text { individual de situação } \\
\text { de dilema } 1 \text { da } \\
\text { situação experimental }\end{array}$ \\
\hline
\end{tabular}

\subsection{Categorização e análise de dados}

A fim de uma melhor compreensão da análise de dados, foram esquematizados os objetivos específicos vinculados diretamente com as diferentes partes do desenho e suas categorizações correspondentes:

Objetivo 1: Comparar o estilo argumentativo dos sujeitos em duas situações: 
EID\&A - Revista Eletrônica de Estudos Integrados em Discurso e Argumentação, Ilhéus, n. 15, jan./jun.2018.

$1^{\text {a }}$ situação: interação (situação experimental)

- em duplas de constituição heterogênea que trabalharam sem assistência

- duplas de constituição heterogênea que trabalharam com assistência do pesquisador (andaimagem dialógica).

$2^{\mathrm{a}}$ situação: argumentação individual escrita (segundo pós-teste).

Objetivo 2: Analisar a competência argumentativa na escrita individual (segundo pós-teste) dos sujeitos que trabalharam em duplas assistidas e não assistidas.

Objetivo 3: Conhecer a melhoria na dialogicidade argumentativa a partir da intervenção do pesquisador em um grupo de duplas em comparação com as duplas que trabalharam sem assistência.

Todas as interações foram gravadas em áudio para sua posterior análise.

O estilo argumentativo foi categorizado em três opções: (a) a posição do falante como resultado da articulação de diferentes pontos de vista; (b) a posição do falante como resultado de uma posição binária; (c) a posição do falante sustentada apenas pela sua própria avaliação dos fatos e a partir da experiência do sujeito (SANTIBÁÑEZ, MIGDALEK e ROSEMBERG, 2016). Cada escrita individual foi categorizada em algum desses estilos. Os argumentos que apareceram de maneira oral durante a interação, por sua vez, foram categorizados em algum desses estilos de modo que durante a interação poderiam ser apresentados diversos estilos argumentativos.

Para avaliar a melhoria na competência argumentativa, foram analisados os escritos individuais dos sujeitos. A competência argumentativa propriamente dita foi conceituada como a capacidade de dar argumentos sobre a própria tese, contra-argumentar, ou seja, de imaginar outras posições diferentes das próprias diante de qualquer situação controvertida e plasmá-las na escrita (CRUZ AGUILAR e CARMONA FERNÁNDEZ, 2014). 
EID\&A - Revista Eletrônica de Estudos Integrados em Discurso e Argumentação, llhéus, n. 15, jan./jun.2018.

Por fim, para testar a melhoria na dialogicidade argumentativa, foram transcritas as interações do primeiro pós-teste (discussão dialógica argumentativa não assistida de situação de dilema 2) e se categorizaram as participações (intervenções dos sujeitos para dar sua opinião sobre a tarefa), avaliações (acordos e desacordos explícitos dos sujeitos a respeito da posição do companheiro) e contra-argumentações (PERALTA, ROSELLI e BORGOBELLO, 2012).

Foi realizada uma contagem de frequência das variáveis mencionadas e foram comparados os resultados obtidos nos dois grupos. Desse modo, podese observar, a partir de um indicador empírico, se a intervenção do pesquisador, cujo objetivo foi andaimar a dialogicidade, teve impacto nos integrantes dessas duplas.

\section{Resultados}

Na Tabela 2, apresentamos uma comparação do estilo argumentativo das duplas em situações de interação, ou seja, as sessões experimentais nas quais os sujeitos interagem em duplas heterogêneas, seja com ou sem assistência do pesquisador.

Tabela 2 - Média do uso do estilo argumentativo em situação experimental (oral) do grupo controle e experimental

\begin{tabular}{llll}
\hline & \multicolumn{4}{l}{ Estilo argumentativo oral (sessão experimental) } \\
\hline & $\mathrm{A}$ & $\mathrm{B}$ & $\mathrm{C}$ \\
\hline $\begin{array}{l}\text { Grupo experimental } \\
\text { (duplas assistidas) }\end{array}$ & - & $M=1.08$ & $M=1.25$ \\
$\begin{array}{l}\text { Grupo controle } \\
\text { (duplas não assistidas) }\end{array}$ & - & $M=1.16$ & $M=1$ \\
\hline
\end{tabular}

As diferenças não são significativas

Como se pode observar, nenhum dos sujeitos apresentou um estilo argumentativo A, ou seja, em seu discurso, não conseguiram integrar diferentes pontos de vistas. No caso do grupo experimental (duplas assistidas), os sujeitos puderam explicitar seus argumentos baseados em suas experiências pessoais e sua própria avaliação dos fatos, enquanto que, durante a interação, os sujeitos do grupo controle (duplas não assistidas) conseguiram expressar, em sua maioria, argumentos baseados em uma posição binária. 
EID\&A - Revista Eletrônica de Estudos Integrados em Discurso e Argumentação, llhéus, n. 15, jan./jun.2018.

Quanto ao estilo argumentativo na escrita individual que os sujeitos realizavam após a interação, pode-se observar que nenhum dos sujeitos conseguiu realizar uma argumentação integradora (tipo A). Os sujeitos do grupo experimental se distribuíram equitativamente entre os dois estilos básicos ( $B=12$ e $C=12$ ), enquanto que os sujeitos do grupo controle apresentaram majoritariamente um estilo binário $(B=18)$.

Em relação à competência argumentativa na escrita individual (segundo pós-teste), dos sujeitos que trabalharam em duplas assistidas e não assistidas, foi possível observar que os sujeitos que passaram por situação de assistência puderam incorporar a contra-argumentação a suas escritas posteriores, enquanto que os sujeitos que não receberam assistência se distribuíram de forma parecida entre o uso e não uso de contra-argumentações (Tabela 3).

Tabela 3 - Competência argumentativa no segundo pós-teste (escrito) dos sujeitos do controle e experimental

\begin{tabular}{lll}
\hline & \multicolumn{2}{l}{$\begin{array}{l}\text { Competência argumentativa - escrito (pós-teste 2: } \\
\text { argumentação individual) }\end{array}$} \\
\hline & Contra-argumentação \\
\hline $\begin{array}{l}\text { Grupo experimental } \\
\text { (duplas assistidas) }\end{array}$ & $62.5 \%$ & NÃO \\
$\begin{array}{l}\text { Grupo controle } \\
\text { (duplas não assistidas) }\end{array}$ & $45 \%$ & $37.5 \%$ \\
\hline
\end{tabular}

A respeito dos aspectos interativos, a melhoria na dialogicidade argumentativa a partir da intervenção do pesquisador avaliada através do primeiro pós-teste alcançou resultados diferentes (Tabela 4).

Tabela 4 - Comparação da dialogicidade na interação livre entre as duplas assistidas e não assistidas

\begin{tabular}{llll}
\hline & \multicolumn{2}{l}{ Dialogicidade (pós-teste 1: discussão não assistida) } \\
\hline & $\mathrm{P}^{*}$ & $\mathrm{CA}$ & $\mathrm{A} / \mathrm{D}^{* * *}$ \\
\hline $\begin{array}{l}\text { Grupo experimental } \\
\text { (duplas assistidas) }\end{array}$ & $\mathrm{M}=12.25$ (D5.13) & $M=2.33$ (D2.42) & $M=3$ (D 1.53) \\
& $M e d=12$ & $M e d=2$ & $M e d=3$ \\
$\begin{array}{l}\text { Grupo controle } \\
\text { (duplas não assistidas) }\end{array}$ & $\mathrm{M}=8.17$ (D6.29) & $M=2.25$ (D 1.21) & $M=5.16$ (D 2.44) \\
\hline
\end{tabular}

* a diferença é estatisticamente significativa $U=37.50 ; p=.045$

** não apresenta diferenças estatisticamente significativas

***a diferença é estatisticamente significativa $U=32.50 ; p=.021$ 
EID\&A - Revista Eletrônica de Estudos Integrados em Discurso e Argumentação, llhéus, n. 15, jan./jun.2018.

Diferenças significativas foram encontradas entre ambos os grupos na participação e avaliação, ainda que em sentidos diferentes. A participação (intervenções dos sujeitos destinadas a expressar opiniões) foi maior no grupo experimental após a assistência quando comparada ao grupo controle, enquanto que a avaliação, ou melhor, a capacidade de expressar acordos e desacordos, foi maior no grupo controle. Pode parecer que a intervenção teve um efeito concreto no que diz respeito a estimular a real participação dos sujeitos nos diálogos, mas ela não conseguiu aumentar a quantidade de intervenções destinadas a avaliar a posição do companheiro em comparação com as duplas do grupo controle. Pode-se estabelecer a hipótese de que a liberdade com que trabalharam no grupo controle permitiu aos sujeitos se posicionarem como avaliadores dos argumentos dos companheiros, enquanto que, nos casos em que houve intervenção, ela parece ter tido o efeito contrário.

Por fim, a contra-argumentação, como capacidade de levantar argumentos alternativos, se manteve nos mesmos níveis em ambos os grupos.

\section{Discussão e conclusões}

A análise da interação social entre os sujeitos constitui um aspecto essencial da pesquisa educativa, especialmente naqueles casos em que se prioriza a colaboração entre pares. Nesse contexto, o estudo da argumentação adquire uma real importância, já que é entendida como um processo psicológico e discursivo que provoca e sustenta raciocínios (MULLER MIRZA e PERRET-CLERMONT, 2009).

Segundo Gutiérrez e Miralba Correa (2008), a argumentação é uma situação de intercâmbio social que permite elucidar características próprias da mente de quem participa de certo intercâmbio, ou seja, permite tornar explícitos os mecanismos envolvidos nessa aprendizagem. Como foi considerada na introdução, a argumentação é, por natureza, conflitiva, e é no seio dessa situação conflitiva que ela se desenvolve em menor ou maior grau.

Pôde-se observar neste estudo que a assistência da argumentação, ou de suas qualidades interativas, tem efeitos benéficos para os sujeitos. Quanto ao estilo, foi detectado que, embora os sujeitos envolvidos não tenham conseguido apresentar um estilo A, quer dizer, o mais complexo que envolve em sua elaboração diferentes pontos de vista, os sujeitos do grupo 
EID\&A - Revista Eletrônica de Estudos Integrados em Discurso e Argumentação, Ilhéus, n. 15, jan./jun.2018.

experimental, após a situação experimental, se distribuíram entre o estilo $B$ e $C$, enquanto que os do grupo controle se concentraram mais em um estilo $B$. Nesse ponto, pode-se observar que a intervenção diversificou os estilos argumentativos presentes. Esse resultado é fundamental para pensar as possíveis aplicações educativas, já que a intervenção não necessariamente envolve um avanço em direção a estilos argumentativos mais complexos, de modo que para cada situação é importante marcar objetivos claros de trabalho. Talvez a natureza da tarefa tenha tido um papel determinante, já que se tratava de situações de dilema que não envolviam conteúdos acadêmicos. Provavelmente, em tarefas acadêmicas, são esperados estilos mais complexos e não posições alternativas como as encontradas neste estudo, ainda que estudos prévios como os de Santibánez (2016) questionem a potencialidade epistêmica da argumentação. Além disso, é possível que a estruturação da tarefa (os sujeitos encontravam-se com duas posições opostas explícitas e os intermédios vinculados a essas posições extremas) pôde ter influenciado diretamente em uma maior quantidade de estilos binários (B).

A respeito da competência argumentativa, pôde-se observar que os sujeitos que passaram por uma situação experimental de assistência conseguiram melhores resultados quanto à presença de contra-argumentação em suas escritas individuais. Talvez este seja o resultado de maior destaque do estudo, já que se sabe que a capacidade de contra-argumentar engloba um conjunto de habilidades necessárias para o desempenho acadêmico dos estudantes na universidade; portanto, se essa capacidade é estimulada nas aulas, é possível obter bons resultados que afetariam positivamente as aprendizagens dos sujeitos.

Por fim, a respeito do estudo da dialogicidade, foi possível observar que os sujeitos que passaram por uma situação de assistência conseguiram ter melhores resultados na participação, ou seja, aumentaram o fluxo de opiniões no que diz respeito à situação levantada, enquanto que a avaliação aumentou nas duplas do grupo controle.

Para concluir, é importante ressaltar a necessidade de incluir em futuros estudos a avaliação de aspectos afetivos na resolução desse tipo de tarefas e replicar este trabalho com sujeitos provenientes de outras disciplinas para comprovar se são encontrados resultados diferentes. 
EID\&A - Revista Eletrônica de Estudos Integrados em Discurso e Argumentação, llhéus, n. 15, jan./jun.2018.

Finalmente, pôde-se constatar que diversos aspectos da competência argumentativa dialógica são suscetíveis de ser melhorados através da argumentação assistida. Esses resultados permitem destacar a importância de estudar a argumentação em contextos colaborativos, em comparação com a argumentação individual e a andaimagem da dialogicidade, como possível melhoria da competência argumentativa tanto dialógica como individual.

\section{Referências}

CHURY, Lady Carolina; ÁLVAREZ HOYOS, Jenny. Desarrolllo de la competencia argumentativa a través de la toma de decisiones en el abordaje de la cuestión sociocientífica: “uso y comercialización del pvc". Revista Góndola, Enseñanza y aprendizaje de las ciencias, v. 10, n.1, p. 56-72, 2015.

CRUZ AGUILAR, María de Lourdes; CARMONA FERNÁNDEZ, Marcelo. Competencias argumentativas en estudiantes de educación superior. Revista Internacional de Ciencias Sociales y Humanidades, v. XXIV, n.2, p.115-137, 2014.

FELTON, Mark; KUHN, Deanna. The development of argumentative discourse skill. Discourse Processes, v. 32, n.2-3, p. 135-153, 2001.

GARCíA-BARRERA, Alba. Importancia de la competencia argumentativa en el ámbito educativo: una propuesta para su enseñanza a través del rol play on line. Revista de Educación a Distancia, v.45, p. 1-21, 2015.

GUTIÉRREZ, Mario Fernando; CORREA, Miralba. Argumentación y concepciones implícitas sobre Física: un análisis pragmadialéctico. Acta Colombiana de Psicología, v. 11, n. 1, p. 55-63, 2008.

GUZMÁN CEDILLO, Yunuen Ixchel; FLORES MACÍAS, Rosa del Carmen; TIRADO SEGURA, Felipe. La evaluación de la competencia argumentativa en foros de discusión en línea a través de rúbricas. Innovación Educativa, v. 12, n.60, p.17-40, 2012.

LEITÃO, Selma. The potential of argument in knowledge building. Human Development, v. 43, n.6, p. 332-360, 2000.

MULLER MIRZA, Nicole; PERRET-CLERMONT, Anne-Nelly. Argumentation and education. Theoretical Foundations and Practices. New York: Springer.

PERALTA, Nadia. Investigar la interacción sociocognitiva en el ámbito educativo: recorrido teórico y resultados empíricos de un estudio en el nivel universitario. Ensemble, v. 4, p.1-14, 2012. 
EID\&A - Revista Eletrônica de Estudos Integrados em Discurso e Argumentação, Ilhéus, n. 15, jan./jun.2018.

PERALTA, Nadia; ROSELLI, Néstor. Modalidad argumentativa en función del tipo de tarea y tamaño del grupo. Cogency, Journal of reasoning and argumentation, v.9, n.2, p.67-83, 2017.

PERALTA, Nadia; ROSELLI, Néstor; BORGOBELLO, Ana. El conflicto sociocognitivo como instrumento de aprendizaje en contextos colaborativos. Interdisciplinaria, v. 29, n. 1, p. 325-338, 2012.

PÉREZ-ECHEVERRíA, María del Puy; POSTIGO, Yolanda; GARCIA-MILA, Merce. Argumentación y educación: apuntes para un debate. Infancia y Aprendizaje, v. 39, n.1, p. 1-24, 2016.

SANTIBÁÑEZ, Cristian. Robustez como categoría para el análisis de la cognición: el caso de la competencia argumentativa. Cinta Moebio, v.52, p. 60-68, 2015.

SANTIBÁÑEZ, Cristian. ¿Para qué sirve argumentar? Problematizando teórica y empíricamente el valor y la función de la argumentación. In C. Santibáñez (Comp.), Ecología Argumentativa Universitaria: Desde la Realidad a los Conceptos, 2016. Concepción: Cosmigonon Ediciones

SANTIBÁÑEZ, Cristian; MIGDALEK, Maia; ROSEMBERG, Celia. Estilos argumentativos en jóvenes universitarios chilenos: Implicancias para una pedagogía colectiva. In C. Santibáñez (Comp.), Ecología Argumentativa Universitaria: Desde la Realidad a los Conceptos, p. 161-186, 2016. Concepción: Cosmigonon Ediciones

Tradução:

Daniel Mazzaro Vilar de Almeida

Docente da Universidade Federal de Uberlândia (UFU)

E-mail: letrasdaniel@yahoo.com.br

Forma de citação sugerida:

PERALTA, Nadia; ROSELLI, Néstor. Estudo quase-experimental sobre argumentação em situações de dilema em diferentes condições de interação. Trad. Daniel Mazzaro Vilar de Almeida. EID\&A - Revista Eletrônica de Estudos Integrados em Discurso e Argumentação, Ilhéus, n. 15, p. 196-208, jan./jun.2018. 